\title{
An atypical case of rare salivary malignancy, hyalinizing clear cell carcinoma
}

\author{
Dong Wook Kim¹, Hye Jeong Park¹, In Ho Cha ${ }^{1,2}$, Dong Hyun Yang ${ }^{2,3}$, Hyun Sil Kim²,3, Woong Nam ${ }^{1,2}$ \\ ${ }^{l}$ Department of Oral and Maxillofacial Surgery, ${ }^{2}$ Oral Cancer Research Institute, \\ ${ }^{3}$ Department of Oral Pathology, College of Dentistry, Yonsei University, Seoul, Korea
}

\begin{abstract}
J Korean Assoc Oral Maxillofac Surg 2013;39:283-288)
As an uncommon, malignant salivary gland tumor with female predominance, hyalinizing clear cell carcinoma (HCCC) is regarded as an indolent tumor. The diagnosis of this rare tumor is challenging, and it depends on microscopic and immunohistochemical (IHC) studies. Although it is regarded as an indolent tumor, there are reports of unconventional forms with aggressive clinical courses. We report an atypical case of this rare tumor, HCCC, in a male patient who had a relatively large-sized mass $(3.8 \times 3.0 \mathrm{~cm})$ on the right mouth floor with ipsilateral neck node metastasis. The clinical, radiological, pathological, and IHC features together with the clinical course are described.
\end{abstract}

Key words: Adenocarcinoma, Clear cell, Sublingual gland, Head and neck neoplasms

[paper submitted 2013. 7. 24 / revised 2013. 9. 16 / accepted 2013. 9. 16]

\section{Introduction}

Hyalinizing clear cell carcinoma (HCCC) is an uncommon, malignant salivary gland tumor ${ }^{1}$ characterized as a distinct entity only recently by Milchgrub et al. ${ }^{2}$ in 1994 . Since then, approximately 40 additional cases have been reported in English literature ${ }^{3}$. HCCC shows female predominance $(72.7 \%)$ and older age in diagnosis (68 years mean), with the vast majority of cases $(81.8 \%)$ occurring in the oral cavity. The most common sites are the base of the tongue, palate, and floor of the mouth; less common locations include the parotid gland, nasopharynx, hypopharynx, and lacrimal gland ${ }^{4}$. Clinically, it is manifested as a small, painless mass that seldom ulcerates ${ }^{3}$. Histologically, all cases of HCCC demonstrate the features

\footnotetext{
Woong Nam

Department of Oral and Maxillofacial Surgery, College of Dentistry, Yonsei University, 50, Yonsei-ro, Seodaemoon-gu, Seoul 120-752, Korea

TEL: +82-2-2228-2971 FAX: +82-2-2227-8022

E-mail: omsnam@yuhs.ac

(C) This is an open-access article distributed under the terms of the Creative Commons Attribution Non-Commercial License (http://creativecommons.org/licenses/by-nc/3.0/), which permits unrestricted non-commercial use, distribution, and reproduction in any medium, provided the original work is properly cited.

Copyright (C) 2013 The Korean Association of Oral and Maxillofacial Surgeons. All rights reserved.
}

of monomorphic glycogen-rich clear cells in cords, nests, islands, or trabeculae within a hyalinized stromal background. A total of 15 cases demonstrated foci of myxoid stromal changes. A dense inflammatory infiltrate consisting of lymphocytes and plasma cells was seen in 5 cases $(9.1 \%)$, with mitotic figures found in 18 cases (32.7\%). O'Regan et al. ${ }^{5}$ reported a case of having aggressive clinical course showing central necrosis with mitotic activity. Perineural invasion was detected in approximately 20 cases (36.4\%), with 1 case showing both perineural and perivascular invasions ${ }^{3}$. Microscopically, the diagnosis presents a challenge due to the heterogeneous nature of the cohort of clear cell salivary gland neoplasms. Thus, several entities should be considered in the differential diagnosis of $\mathrm{HCCC}^{1,2}$, including mucoepidermoid, epithelial-myoepithelial, acinic cell, and metastatic carcinomas $^{1,2}$.

We report an atypical case of this rare tumor, HCCC, in a male patient who had a relatively large-sized mass $(3.8 \times 3.0$ $\mathrm{cm})$ on the right mouth floor with ipsilateral neck node metastasis. The neck node was rare not only in terms of its presence but also its relatively large size $(3.0 \mathrm{~cm})$. The clinical, radiological, pathological, and immunohistochemical (IHC) features together with the clinical course are described.

This work was supported by the Priority Research Centers Program through the National Research Foundation of Korea (NRF) funded by the Ministry of Education, Science, and Technology (2009-0094027). 


\section{Case Report}

In December 2012, a 54-year-old male patient visited our clinic complaining of painless sublingual swelling on the

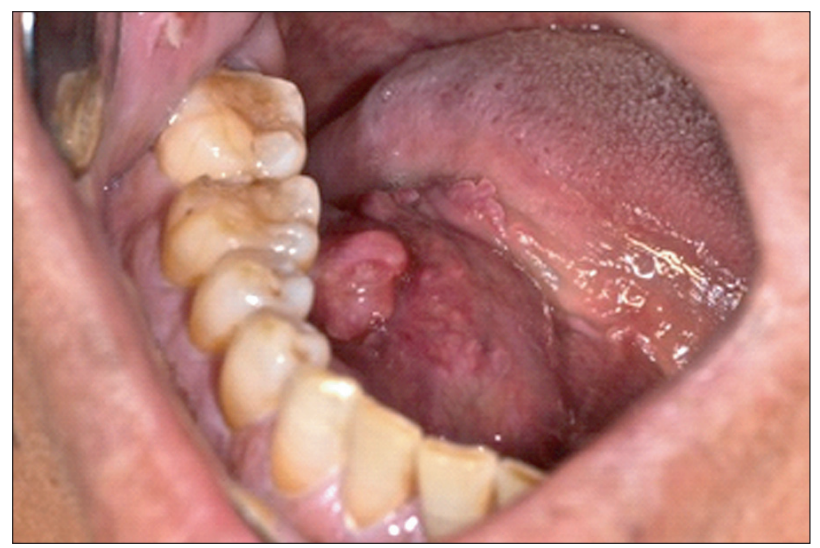

Fig. 1. Initial intraoral photograph.

Dong Wook Kim et al: An atypical case of rare salivary malignancy, hyalinizing clear cell carcinoma. J Korean Assoc Oral Maxillofac Surg 2013 right side, which he had first noticed 2 weeks earlier. His medical and family histories were unremarkable. Upon physical examination, a smooth, non-tender, firm, approximately $4 \mathrm{~cm}$ mass was detected on the right floor of the mouth. It had a $0.5 \mathrm{~cm}$ pedunculated exophytic mass on the top of its dome-shaped surface.(Fig. 1) There was neither limitation nor deviation of tongue movement nor paresthesia on the oral and facial regions. He also had a $2 \mathrm{~cm}$ palpable lymph node on the ipsilateral neck, level II, which was round, non-tender, firm, and fixed to adjacent structures.

The gadolinium-enhanced magnetic resonance imaging (MRI) showed a $4 \mathrm{~cm}$ enhancing lesion on the right floor of the mouth and sublingual space, with signs of possible invasion into the right mylohyoid muscle.(Fig. 2. A) There was no evidence of midline crossing or bone invasion. Nodal metastasis in the right level II was also noted.(Fig. 2. B) Positron emission tomography-computed tomography (PET-CT) showed fluorine-18-fluorodeoxyglucose (18F-FDG) uptake in the right sublingual space, suggesting probable malignancy.
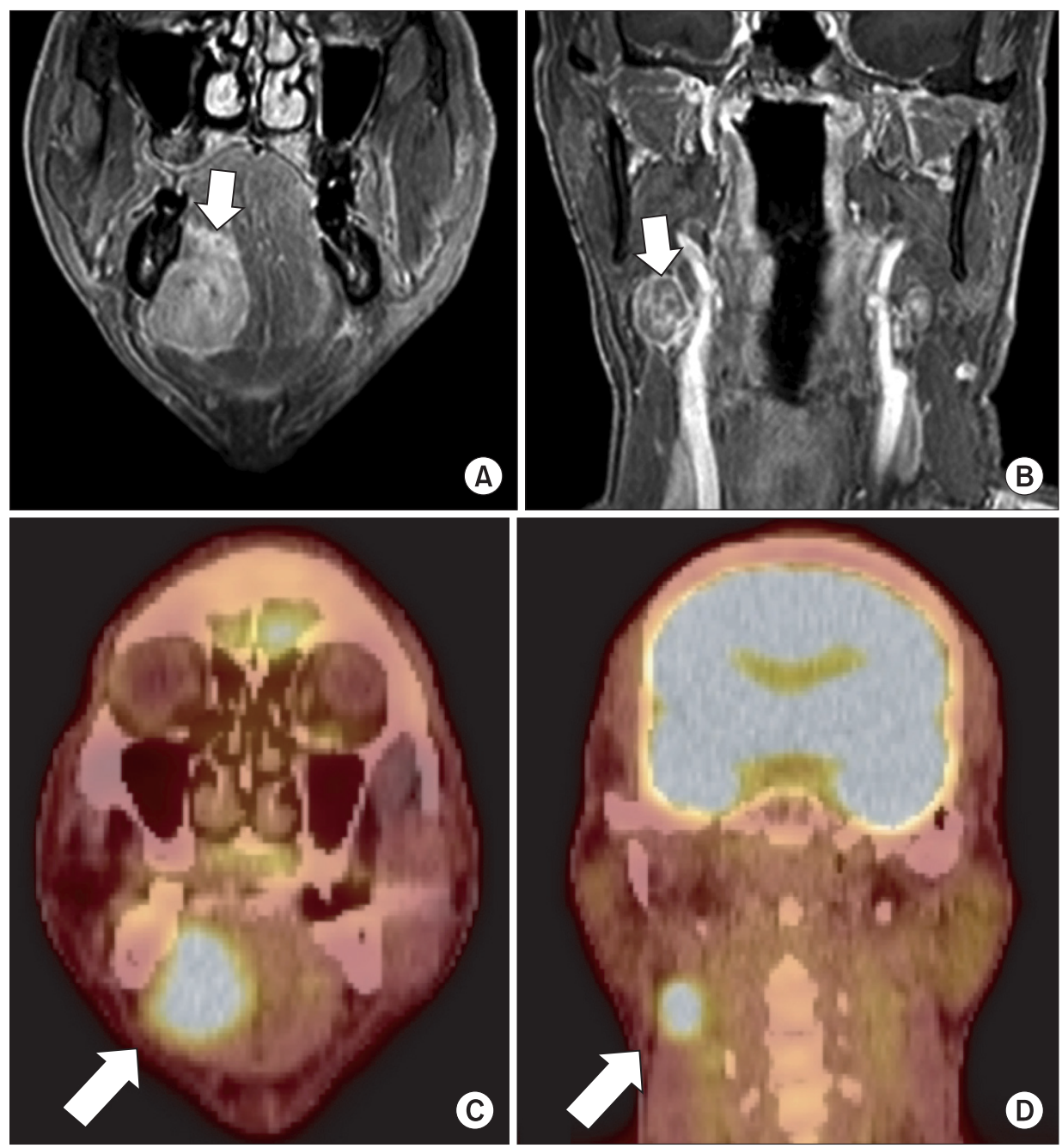

Fig. 2. Gadolinium-enhanced magnetic resonance imaging, coronal view. A. Main mass (arrow). B. Nodal metastasis on level II, ipsilateral side (arrow). C. Positron emission tomography-computed tomography, coronal view. Main mass (arrow). D. Nodal metastasis on level II, ipsilateral side (arrow).

Dong Wook Kim et al: An atypical case of rare salivary malignancy, hyalinizing clear cell carcinoma. $J$ Korean Assoc Oral Maxillofac Surg 2013 
(Fig. 2. C) Enlarged lymph node with 18F-FDG uptake was also observed in the level II of ipsilateral neck, suggesting probable metastatic lymph node.(Fig. 2. D)

The microscopic examination of incisional biopsy showed poorly differentiated epithelial cells with multiple clear cells. (Fig. 3. A, 3. B) The specimen was mostly composed of malignant cells with clear cytoplasm, with some portions of cells showing glandular differentiation. Mucicarmine staining was performed to rule out mucoepidermoid carcinoma. The cells of the specimen were negative to mucicarmine staining; thus ruling out mucoepidermoid carcinoma.(Fig. 3. C) Based on these results, the pathologists suggested--in the preliminary report of incisional biopsy--that the tumor was malignant epi- thelial tumor or metastatic tumor from kidney.

IHC studies with S-100, cytokeratin 7 (CK7), and CK20 were performed during further diagnosis. S-100 was for myoepithelial carcinoma, showing immunoreactivity for S-100 in $100 \%$ of cases ${ }^{6}$. CK7 and CK20 were performed to evaluate the possibility of metastatic carcinoma because clear cells containing tumor in the salivary gland can be metastatic, as in the case of renal cell carcinoma ${ }^{7}$. In this case, the tumor cells of incisional biopsy revealed no reactivity to S-100 (Fig. 3. D); thus excluding the possibility of myoepithelial carcinoma. It tested positive to CK7 (Fig. 3. E) but negative to CK20.(Fig. 3. F) This CK(+)/CK20(-) immunophenotype in salivary gland tumor allowed the exclusion of metastatic
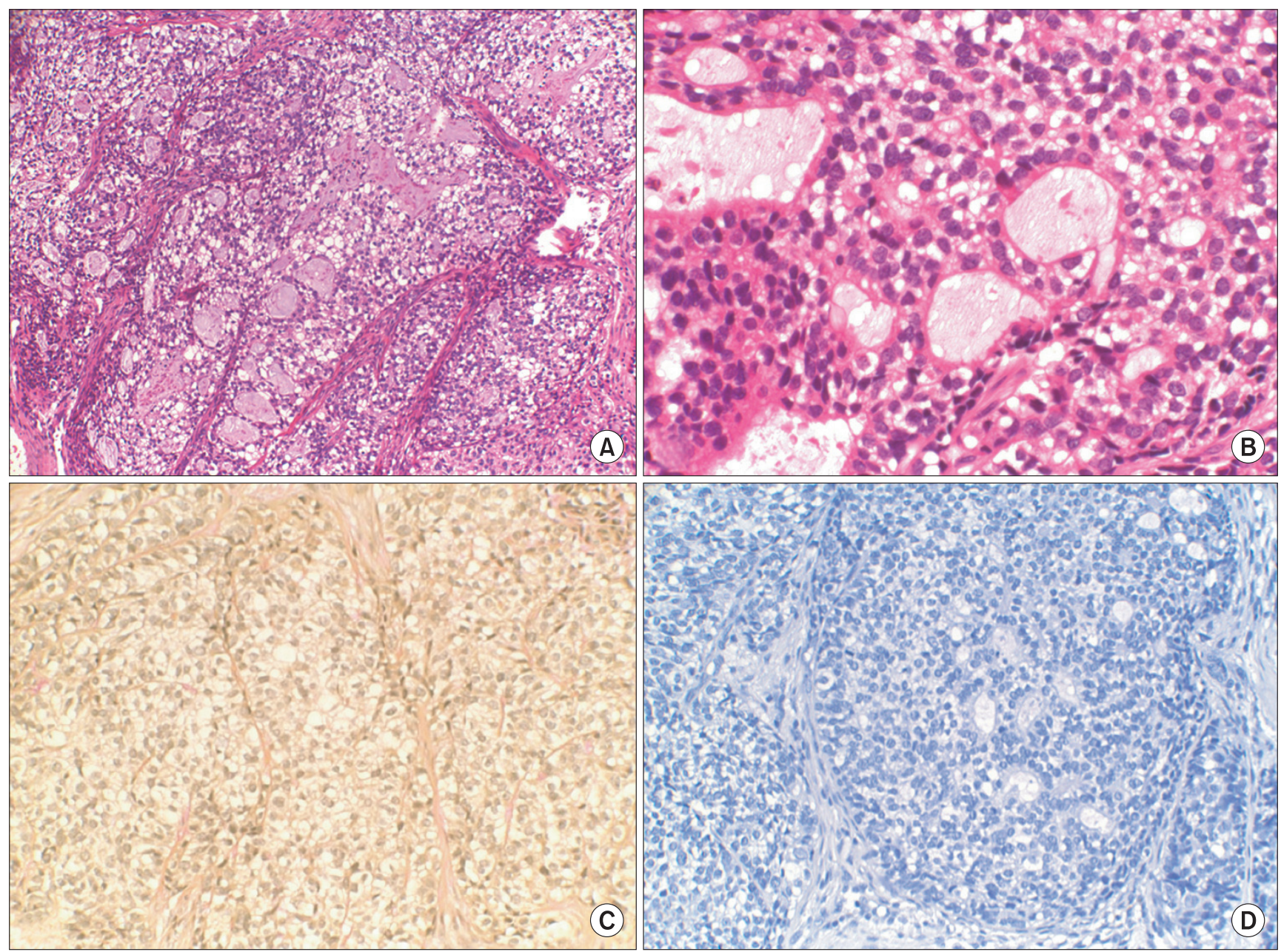

Fig. 3. A. Histopathological examination of incisional biopsy. Poorly differentiated epithelial cells with multiple clear cells were noted $(H \& E$ staining, x100). B. Specimen from incisional biopsy in higher magnification. The specimen was mostly composed of malignant cells with clear cytoplasm, and there were some portions of cells with glandular differentiation (H\&E staining, x400). C. The specimen from incisional biopsy showing clear cells stained negatively to mucicarmine (x200). D. Immunohistochemical (IHC) staining for S-100 showing negative reactivity of clear cells (x200). E. IHC staining for cytokeratin (CK) 7 showing positive reactivity of clear cells (x200). F. IHC staining for CK20 showing negative reactivity of clear cells (x200). G. Histopathologic examination of final specimen. Epithelial cells and clear cells arranged in cords or nests (H\&E staining, x100). H. Final specimen in higher magnification. Areas of hyalinization are seen (marked in asterisks) (H\&E staining, $x 400)$.

Dong Wook Kim et al: An atypical case of rare salivary malignancy, hyalinizing clear cell carcinoma. J Korean Assoc Oral Maxillofac Surg 2013 

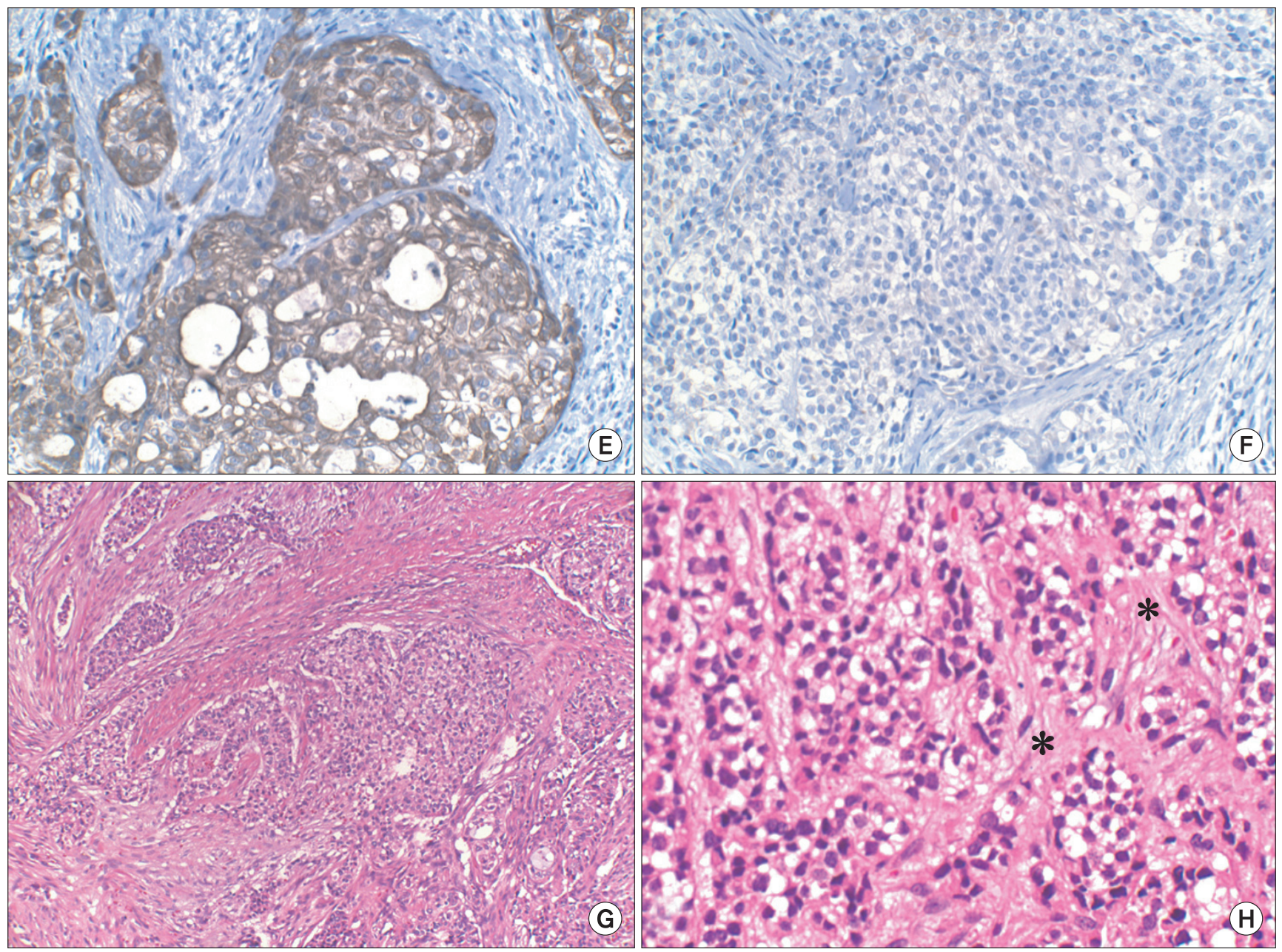

Fig. 3. Continued.

Dong Wook Kim et al: An atypical case of rare salivary malignancy, hyalinizing clear cell carcinoma. J Korean Assoc Oral Maxillofac Surg 2013

carcinomas such as renal cell carcinoma or malignant melanoma $^{7}$. The pathologist suggested that the tumor was acinic cell carcinoma or adenocarcinoma--not otherwise specified-unless it is not metastatic carcinoma.

In the operating room, modified radical neck dissection together with the preservation of the spinal accessory nerve, internal jugular vein, and sternocleidomastoid muscle was performed on the ipsilateral neck, including selective neck dissection of contralateral level IA.(Fig. 4) Wide excision of the main mass measuring $5.0 \times 4.0 \times 3.5 \mathrm{~cm}$ was done, with its section revealing a partly necrotic, yellowish white cut surface measuring $3.8 \times 3.0 \mathrm{~cm}$.

The microscopic examination of the final specimen showed clear cells and epithelial cells arranged in cords or nests in connective tissue (Fig. 3. G), with areas of hyalinization.(Fig. 3. H) Considering the results of special staining and IHC studies performed on incisional biopsy, these features were consistent with the HCCC of salivary gland. Additionally, there were frequent lymphovascular permeations. Note, however, that mitoses were not frequent, and perineural invasion was not identified. Resection margins were free of carcinoma, with the margin of clearance being $100 \mu \mathrm{m}$. The lymph node from level II of the right neck, which had hot uptake on PET-CT, showed metastatic adenocarcinoma on frozen biopsy and final specimen.

The patient was referred to the department of radiation oncology for postoperative radiation therapy. He received a total of 60 Gy for 6 weeks of image-guided, intensitymodulated radiation therapy. He completed the radiotherapy without complications other than mild oral mucositis, and he is showing good progress. There was no evidence of recurrence upon follow-up examination and imaging studies. MRI taken 6 months after the surgery showed no residual or recurred tumor. PET-CT taken 8 months after the surgery did 

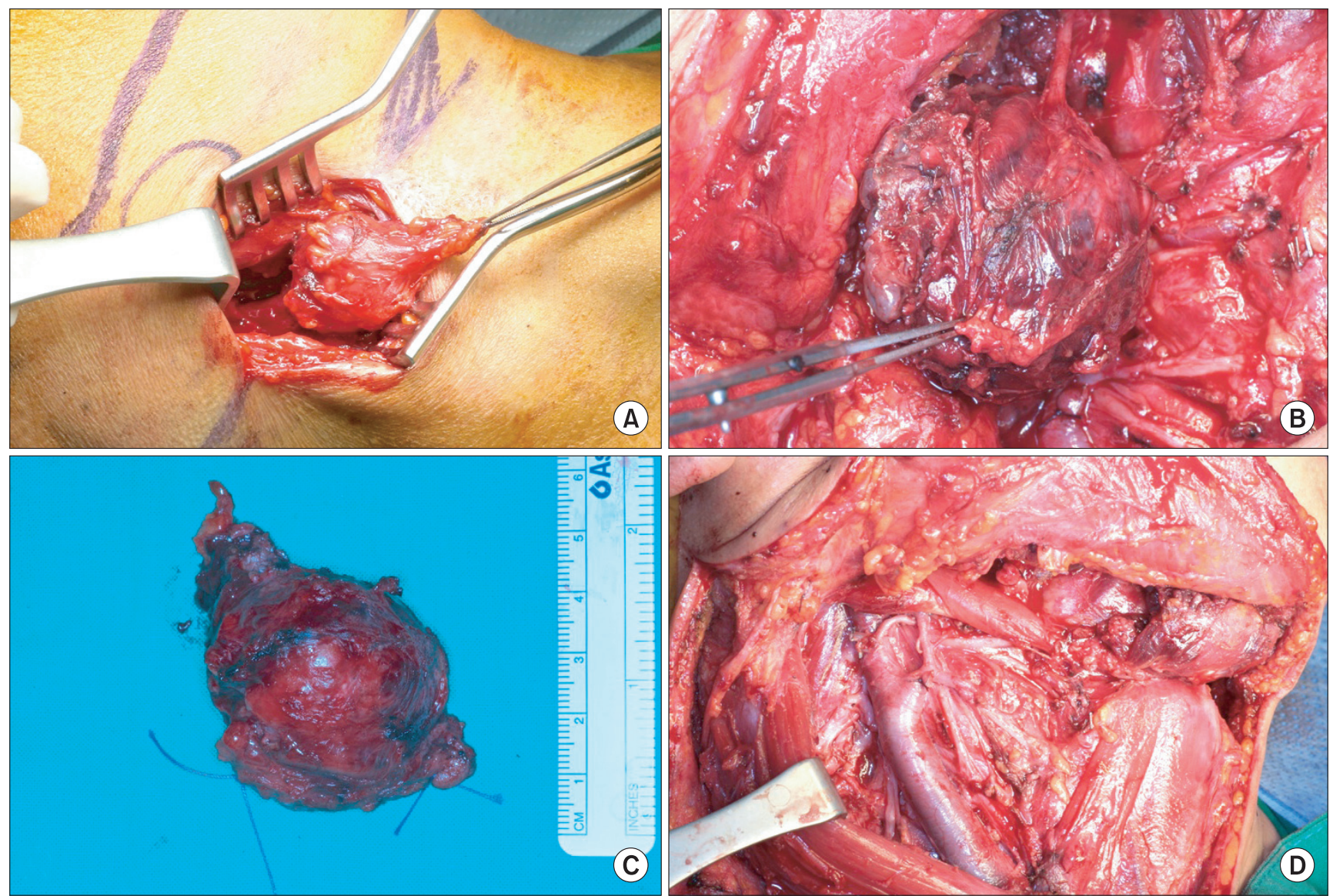

Fig. 4. Intraoperative photographs. A. Palpable node on level II was revealed. B. The main lesion was shown. C. Surgical specimen of the main mass. D. Operation site after the excision and neck dissection.

Dong Wook Kim et al: An atypical case of rare salivary malignancy, hyalinizing clear cell carcinoma. J Korean Assoc Oral Maxillofac Surg 2013

not show any discernible 18F-FDG uptake to suggest tumor recurrence.

\section{Discussion}

Clear cells create diagnostic dilemmas and controversies in the classification of clear cell neoplasms ${ }^{3}$. Salivary gland neoplasms associated with a high proportion of clear cells include clear cell mucoepidermoid carcinoma, epithelialmyoepithelial carcinoma, acinic cell carcinoma, some odontogenic tumors, and metastatic clear cell tumors ${ }^{1,2}$. In most cases, clear cells make up only a minor portion, with the appropriate classification of the tumor being relatively easy on the basis of typical features. In some tumors, however, they comprise a major portion, making diagnosis highly challeng$\mathrm{ing}^{8}$. It is important to differentiate HCCC from other tumors with clear cells since their treatment and clinical outcome are different.

Histopathological examination and IHC testing are known bases for differentiating types of tumors containing clear cells $^{3}$. In clinical practice, the diagnostic term 'clear cell carcinoma' is a diagnosis of exclusion, applied only after the exclusion of other specific tumors with clear cell morphology ${ }^{3}$.

In this case, the negative result of mucicarmine staining ruled out the possibility of mucoepidermoid carcinoma. Acinic cell carcinoma was excluded since it is often infiltrative, showing different growth patterns including solid, papillary, microcystic, and follicular patterns and containing more than one cell type. Clear cells are occasionally numerous but rarely predominate. These tumors stain positively with mucicarmine?

Immunohistochemistry was helpful in revealing the origin of tumor and differentiating the diagnosis. Myoepithelial carcinoma could be excluded by the negative result of IHC study with S-100, since it shows immunoreactivity for S-100 protein and vimentin in $100 \%$ of cases. It is variably associated with immunolabeling for CK7, CK14, smooth muscla actin, and calponin ${ }^{6}$. Clear cell odontogenic carcinoma is an 
aggressive tumor and is morphologically characterized by islands of mostly clear cells surrounded by fibrous stroma. Tumor cells possess abundant glycogen and test positive for cytokeratin and epithelial membrane antigen. Unlike HCCC, however, clear cell odontogenic carcinoma tends to be S-100 positive $^{4}$.

The differential expression of CK7 and 20 in carcinomas facilitates differentiation of primary salivary gland neoplasms from metastatic tumors and squamous carcinoma ${ }^{7}$. CK7 positive and CK20 negative results excluded the possibility of metastatic carcinoma and confirmed that this tumor have originated with salivary gland.

This case is unique from other reported cases because it is known to occur mostly in females ${ }^{2}$ with reported size range of $0.3-3.0 \mathrm{~cm}^{10}$. In this case, the patient was male, and the size was $3.8 \times 3.0 \mathrm{~cm}$, which is just outside that range. Moreover, the patient had lymph node metastasis, also a rare symptom.

HCCC is regarded as an indolent tumor. At least 2 of the 11 patients reported by Milchgrub et al. ${ }^{2}$ had metastases to cervical lymph nodes at the time of clinical manifestation, but none developed local or distant metastases. Nonetheless, recurrences and metastases have been occasionally reported in $\mathrm{HCCC}^{5,11}$. Tang et al. ${ }^{11}$ described an HCCC occurring in the nasopharynx and which recurred on three occasions, and it was probably related to the difficulty in achieving complete excision. In 2000, Ereño et al. ${ }^{12}$ reported an HCCC that developed distant metastases in the form of multiple right lung basal lobe metastases, with a fine needle aspirate of these lesions showing abundant clear cells. O'Regan et al. ${ }^{5}$ reported an aggressive form of HCCC that showed a course of widespread metastases and death within a year of initial manifestation in a female patient. They reported that, initially, the mass was on the right side of the tongue, extending to the right parapharyngeal space along with an enlarged lymph node on the right side of her neck. She underwent radical surgery including neck dissections and postoperative radiation therapy. The pathologic examination showed minor foci of mitotic activity, necrosis, anaplasia, and extensive nodal involvement. They suggested that there may be a subset of these tumors with a more aggressive clinical course than previously known, proposing that minor foci with mitotic activity and pleomorphism might be related ${ }^{5}$. In this case, there were no notable foci of mitotic activities and pleomorphisms.

There are no proven prognostic factors of HCCC, a rare tumor. Our case appears to be an atypical instance of this rare tumor considering the gender of the patient, size of the mass, and lymph node metastasis. Further follow-up of this patient may yield insights into the prognostic factors and treatment planning as well as better understanding of this rare entity of salivary malignancy.

\section{References}

1. Dardick I, Leong I. Clear cell carcinoma: review of its histomorphogenesis and classification as a squamous cell lesion. Oral Surg Oral Med Oral Pathol Oral Radiol Endod 2009;108:399-405.

2. Milchgrub S, Gnepp DR, Vuitch F, Delgado R, Albores-Saavedra J. Hyalinizing clear cell carcinoma of salivary gland. Am J Surg Pathol 1994;18:74-82.

3. Baghirath PV, Kumar JV, Vinay BH. Hyalinizing clear cell carcinoma: a rare entity. J Oral Maxillofac Pathol 2011;15:335-9.

4. O'Sullivan-Mejia ED, Massey HD, Faquin WC, Powers CN. Hyalinizing clear cell carcinoma: report of eight cases and a review of literature. Head Neck Pathol 2009;3:179-85.

5. O'Regan E, Shandilya M, Gnepp DR, Timon C, Toner M. Hyalinizing clear cell carcinoma of salivary gland: an aggressive variant. Oral Oncol 2004;40:348-52.

6. Savera AT, Sloman A, Huvos AG, Klimstra DS. Myoepithelial carcinoma of the salivary glands: a clinicopathologic study of 25 patients. Am J Surg Pathol 2000;24:761-74.

7. Meer S, Altini M. CK7+/CK20- immunoexpression profile is typical of salivary gland neoplasia. Histopathology 2007;51:26-32.

8. Ellis GL. Clear cell neoplasmsin salivary glands: clearly a diagnostic challenge. Ann Diagn Pathol 1998;2:61-78.

9. Said-Al-Naief N, Klein MJ. Clear cell entities of the head and neck: a selective review of clear cell tumors of the salivary glands. Head Neck Pathol 2008;2:111-5.

10. Batsakis JG, el-Naggar AK, Luna MA. Hyalinizing clear cell carcinoma of salivary origin. Ann Otol Rhinol Laryngol 1994;103:7468.

11. Tang SK, Wan SK, Chan JK. Hyalinizing clear cell carcinoma of salivary gland: report of a case with multiple recurrences over 12 years. Am J Surg Pathol 1995;19:240-1.

12. Ereño C, Grande J, Alija V, Yarnoz J, Bilbao FJ. Hyalinizing clear cell carcinoma of the hypopharynx metastasizing to the lung: a case report. Histopathology 2000;37:89-91. 